\title{
Lattice and beam parameters of compact intense $x$-ray sources based on Compton scattering
}

\author{
Peter Gladkikh* \\ National Science Center, Kharkov Institute of Physics and Technology, Kharkov, Ukraine
}

(Received 13 August 2004; published 18 May 2005)

\begin{abstract}
The lattice of a laser-electron storage ring with a controlled momentum compaction factor dedicated to the generation of intense $\mathrm{x}$ rays under Compton scattering is described. In such a storage ring one can obtain large energy acceptance and keep the long-term stable motion of the electron beam with large energy spread. The intensity of $\mathrm{x}$ rays is stable due to the use of electron beam with steady-state parameters for Compton scattering. Parameters of the storage ring, electron beam, and x rays obtained by electron beam dynamics simulation involving both intrabeam and Compton scattering are presented.
\end{abstract}

DOI: 10.1103/PhysRevSTAB.8.050702

PACS numbers: 41.60. $-\mathrm{m}, 29.27 . \mathrm{Bd}, 29.20 . \mathrm{Dh}$

\section{INTRODUCTION}

The proposal of the laser-electron storage rings (LESR) dedicated to generation of hard $\mathrm{x}$ rays by means of the Compton scattering (CS) of the laser light on low-energy electron beam of the storage ring was stated in 1998 [1]. Under scattering in head-on collision the quanta with maximal energy $\varepsilon_{\gamma \max }=4 \gamma^{2} \varepsilon_{\text {las }}$ are scattered towards the direction of electron velocity, where $\varepsilon_{\text {las }}$ is the laser photon energy and $\gamma$ the Lorentz factor. For example, to obtain X-ray energy $\varepsilon_{\gamma} \approx 33 \mathrm{keV}$ for angiographic studies under Compton scattering of laser photons with energy $\varepsilon_{\text {las }}=1.164 \mathrm{eV}$ (neodymium laser) one needs electron beam energy $E_{0} \approx 43 \mathrm{MeV}$. To generate such $\mathrm{x}$ rays in the synchrotron radiation (SR) source one needs the electron beam energy $E_{0} \approx 2.5 \mathrm{GeV}$ and a superconducting wiggler with field $B_{0} \approx 7.5 \mathrm{~T}$. It is evident that x-ray generators based on CS may become inexpensive, compact sources of the intense $x$ rays.

There are two main problems in LESR design. The first one is associated with the strong effect of intrabeam scattering (IBS) at low electron beam energy. Because of this effect, the beam size grows quickly and CS intensity decreases. The second problem is associated with large electron beam energy spread because of the fluctuation of Compton generation. The value of the energy spread will reach a few percent, and in these conditions one needs to keep the electron beam during long term in order to achieve the high Compton beam intensity.

Two basic schemes of LESR were proposed for the generation of intense Compton beams. In the first scheme, the electron beam with non-steady-state parameters is supposed to be used [2]. The intense electron beam is injected into the storage ring and this beam is being used during short term, within which the beam size does not significantly increase, and after that injection is repeated. For such experiments one needs a very intense, lowemittance injector. Under developing parameters of the laser systems designers expect an average scattered beam

*Electronic address: gladkikh@kipt.kharkov.ua intensity $n_{\gamma} \approx 10^{13} \mathrm{phot} / \mathrm{s}$ and spectral brightness $B \approx$ $10^{13} \mathrm{phot} /\left(\mathrm{s} \mathrm{mm}^{2} \mathrm{mrad} 0.1 \% \mathrm{BW}\right)$. The main disadvantage of such a LESR scheme is the pulsed nature of radiation, whereas some experiments require long-term stability of x-ray intensity (for example, biological studies, laser cooling of electron beam, etc.).

In this paper the second scheme of LESR with a controlled momentum compaction factor $[3,4]$ is described. In such a storage ring one can achieve the large energy acceptance and keep the long-term stable motion of electron beam with large energy spread. The intensity of $x$ rays is stable due to the use of an electron beam with steadystate parameters. The computed parameters of $\mathrm{x}$ rays are presented. All dependencies were obtained by simulation of the electron beam dynamics involving both IBS and CS.

\section{MAIN REQUIREMENTS FOR RING LATTICE}

The partial energy spread $\delta_{\mathrm{CS}}$ caused by fluctuation of $\mathrm{CS}$ is much greater than the energy spread $\delta_{\mathrm{SR}}$ of SR. It is associated with large energies of the quanta generated at $\mathrm{CS}$. The value of $\delta_{\mathrm{CS}}$ is described by the expression [5]

$$
\delta_{\mathrm{CS}}=\sqrt{\frac{2}{3} \gamma \frac{\varepsilon_{\mathrm{las}}}{\varepsilon_{0}}}
$$

where $\varepsilon_{0}$ is the electron rest energy. Under intense CS when energy losses $\Delta E_{\mathrm{CS}}$ caused by CS are comparable to those $\Delta E_{\mathrm{SR}}$ of $\mathrm{SR}$, the value of the steady-state energy spread $\delta_{\text {tot }}$ reaches a few percent [5]

$$
\delta_{\mathrm{tot}}=\sqrt{\frac{\Delta E_{\mathrm{CS}}}{\Delta E_{\mathrm{tot}}} \delta_{\mathrm{CS}}^{2}+\frac{\Delta E_{\mathrm{SR}}}{\Delta E_{\mathrm{tot}}} \delta_{\mathrm{SR}}^{2}} \approx \sqrt{\frac{\Delta E_{\mathrm{CS}}}{\Delta E_{\mathrm{tot}}}} \delta_{\mathrm{CS}},
$$

where $\Delta E_{\mathrm{tot}}=\Delta E_{\mathrm{SR}}+\Delta E_{\mathrm{CS}}$ are the total energy losses. To obtain the stable electron beam motion in these conditions, we need to solve several serious problems.

\section{A. Quantum lifetime}

To obtain the sufficient quantum lifetime, we need the large energy acceptance of the storage ring $\sigma_{\text {rf }}$, and un- 
reasonably high rf voltage $V_{\text {rf }}$ may be required because $\sigma_{\text {rf }} \propto \sqrt{V_{\text {rf }} / \alpha_{1}}$, where $\alpha_{1}$ is the momentum compaction factor of the first order. There is no space in a compact storage ring for the placement of the number of rf cavities. The less the momentum compaction factor, the greater the energy acceptance, so it is needed to design ring lattice with the minimal linear momentum compaction factor.

\section{B. Chromatic effects}

Transversal and longitudinal beam dynamics are determined at large energy spread not only by linear on momentum deviation effects, but also by quadratic and higher order ones. The displacement of the electron orbit $\Delta x_{C}$ from the reference one is determined, as it is well known, by dispersion functions

$$
\Delta x_{C}=\eta_{1} \delta+\eta_{2} \delta^{2}+\ldots,
$$

where $\eta_{1}, \eta_{2}$ are the first and second order dispersion functions, correspondingly, and $\delta=\Delta p / p$ is the momentum deviation. In the laboratory frame, under the assumption of the Gaussian distribution of densities of electron and laser beams, the number of scattered photons generated in single collision is determined by the following expression [6]:

$$
n_{\gamma}=\frac{n_{e} n_{\mathrm{las}} \sigma}{2 \pi \sqrt{\sigma_{z e}^{2}+\sigma_{z l}^{2}} \sqrt{\left(\sigma_{x e}^{2}+\sigma_{x l}^{2}\right)+\left(\sigma_{s e}^{2}+\sigma_{s l}^{2}\right) \tan ^{2}(\varphi / 2)}},
$$

where $\sigma_{z e}$ and $\sigma_{z l}$ are the vertical sizes of electron and laser beams, $\sigma_{x e}$ and $\sigma_{x l}$ the horizontal sizes of colliding beams, $\sigma_{s e}$ and $\sigma_{s l}$ the longitudinal ones, $\varphi$ the collision angle ( $\varphi=0$ corresponds to head-on collision), $n_{e}$ and $n_{\text {las }}$ the number of particles in colliding bunches, and $\sigma$ the total cross section of Thomson scattering; here and below we suggest the electron and laser beams colliding in the horizontal plane.

The dispersions cause the growth of the effective beam emittance and, consequently, the decreasing of the CS intensity, so both dispersions should be suppressed at the interaction point (IP). The suppression of the first order dispersion is only performed by using of the linear elements of lattice (bendings and quadrupoles). To suppress the second order dispersion we also need to phase the sextupoles placed over ring circuference with the nonzero dispersion of the first order $[7,8]$.

In the case of a non-head-on collision, the intensity of the CS significantly depends on the longitudinal size of the electron beam as is evident from (4), because usually $\sigma_{s e}>\sigma_{s l}$. To obtain the high intensity of CS, a lattice with low momentum compaction factor $\alpha_{1}$ is needed, because the longitudinal size $\sigma_{s e} \propto \sqrt{\alpha_{1}}$. Besides, the small collision angle is also needed for intensive CS at non-head-on collision.
Betatron frequencies and amplitude functions also depend on momentum deviation because of the changing of the strengths of lattice elements. These dependencies are characterized by derivatives (see, e.g., [7]) $\partial Q_{x, z} / \partial \delta, \partial^{2} Q_{x, z} / \partial \delta^{2}, \ldots$ The changes in betatron amplitudes and frequencies cause nonlinearity in the betatron motion. In this connection the effective emittance increases. Besides, the nonlinear dependence of the betatron tuning on momentum deviation can stimulate betatron resonances even if natural chromaticity is compensated: $\partial Q_{x, z} / \partial \delta=0$.

In the case of both large momentum deviation and second order dispersion, the quadratic term of the transversal displacement (3) becomes comparable to the linear one. This effect causes quadratic terms in orbit lengthening, in other words, the momentum compaction factor becomes dependent on the momentum deviation $[9,10]$

$$
\alpha=\alpha_{1}+\alpha_{2} \delta+\ldots,
$$

where $\alpha_{2}$ is the momentum compaction factor of the second order.

The quadratic on momentum deviation terms become apparent in longitudinal dynamics as the distortion of the separatrix shape and reduction of the rf acceptance. Thus, the possibilities of the suppression of aberrations are needed in ring lattice.

\section{Intrabeam scattering}

The effect of the IBS becomes very strong at low electron beam energies. The emittance growth comparably to a natural emittance can reach 2-3 orders; it causes the essential increasing of the beam size and significant decreasing of the CS intensity. It is a very complicated analytical task to take into account the IBS involving CS because the problem is self-consistent. We simulated the IBS by using of the following algorithm:

(i) The growth rates $\tau_{y}, y=\{x, z, s\}$ are computed by [11] before particle tracking over estimated range of the beam emittances.

(ii) The IBS element is incorporated in ring lattice, and angular coordinates and momentum deviation are changed in this element by the use of matrix transformation

$$
y_{\text {fin }}^{\prime}=y_{\text {ini }}^{\prime}\left(1+T_{\text {rev }} / \tau_{y}\right), y^{\prime}=\left\{x^{\prime}, z^{\prime}, \delta\right\},
$$

where $T_{\text {rev }}$ is the revolution time.

During simulation the growth rates are corrected in accordance to beam emittances. If the correction time is much smaller than growth rates, we obtain the steady-state emittances coinciding well with analytical estimations.

\section{Dynamics aperture}

Sextupole lenses are used in storage rings to correct chromatic effects. The natural chromaticity of the storage ring is very large under the condition of the strong focusing of the electron beam at IP and the required sextupole 
strengths also become large in a compact storage ring with low- $\beta$ insertion. A dynamics aperture of the ring (DA) is reduced and the problem of obtaining the acceptable DA should also be solved at lattice design.

\section{RING LATTICE}

In order to meet the above described requirements, the LESR lattice with controlled momentum compaction factor is proposed for $\mathrm{x}$-ray generator NESTOR designed at NSC KIPT. Its layout is presented in Fig. 1.

The storage ring is of the racetrack type. The long straight section with IP is dispersion free while the dispersion of the opposite long straight section is nonzero. Separated quadrupole lenses in ring arcs perform the control of the momentum compaction factor. Quadrupole quadruplets on a long straight section focus the electron beam at IP. About only one-third of the ring orbit is dispersion free, and the long dispersion section allows the placing of the great number of sextupole lenses for correction of the chromatic effects. The strong sextupoles effect on beam dynamics similarly octupoles; to correct such effect four combined sextupole lenses with octupole field component are incorporated in ring lattice. The rf cavity is placed on a dispersion free IP-drift; the injection system is placed on the opposite drift. The maximal length of the drift spaces is approximately equal to $1.2 \mathrm{~m}$, and it allows one to place the $700 \mathrm{MHz}$ rf cavity and injection system elements on those drifts. The ring circumference is equal to $15.418 \mathrm{~m}$ (harmonics number $h=36$ ).
A ferrite kicker magnet placed external to the ceramic vacuum chamber with thin metal deposition will be used to inject the electron beam in the horizontal plane on-axes into the storage ring. Injection on full energy will be realized at the electron beam energy $E_{0} \leq 60 \mathrm{MeV}$, adiabatic raising of the electron beam energy in storage ring will be used at operation modes with $E_{0}>60 \mathrm{MeV}$. A kicker is placed at the azimuthal position with the horizontal amplitude function close to its maximal value over the ring. Kicker field strength is about $3.5^{\circ}$ (kicker field $B_{k} \approx 0.02 \mathrm{~T}$ at the ferrite length $L_{k}=0.6 \mathrm{~m}$ at electron energy $E_{0}=60 \mathrm{MeV}$ ). The repetition rate of the electron bunches $f_{\text {rep }}=350 \mathrm{MH} z$, consequently, it requires the back pulse edge $\tau_{\text {bpe }} \leqslant 3$ ns. Such pulse parameters are realizable at deposit thickness $t_{d} \lesssim 3 \mu$. The electron beam will be transported from an injection channel to a kicker through fringing field of the bending using a shielding magnet channel.

The amplitude functions over a half of the ring lattice are presented in Fig. 2 (ring is reflection symmetric to interaction point; curves begin from IP). Different focusing of the IP drift and the opposite one (because dispersion functions over these drifts are different) causes an insignificant asymmetry of the amplitude functions over arc and long straight sections. The less the $\beta$-function asymmetry, the less the amplitudes of the azimuthal perturbation harmonics, and the greater the dynamics aperture of the ring.

Both the first and the second order dispersion functions $\eta_{1}$ and $\eta_{2}$ over half of the ring lattice are presented in

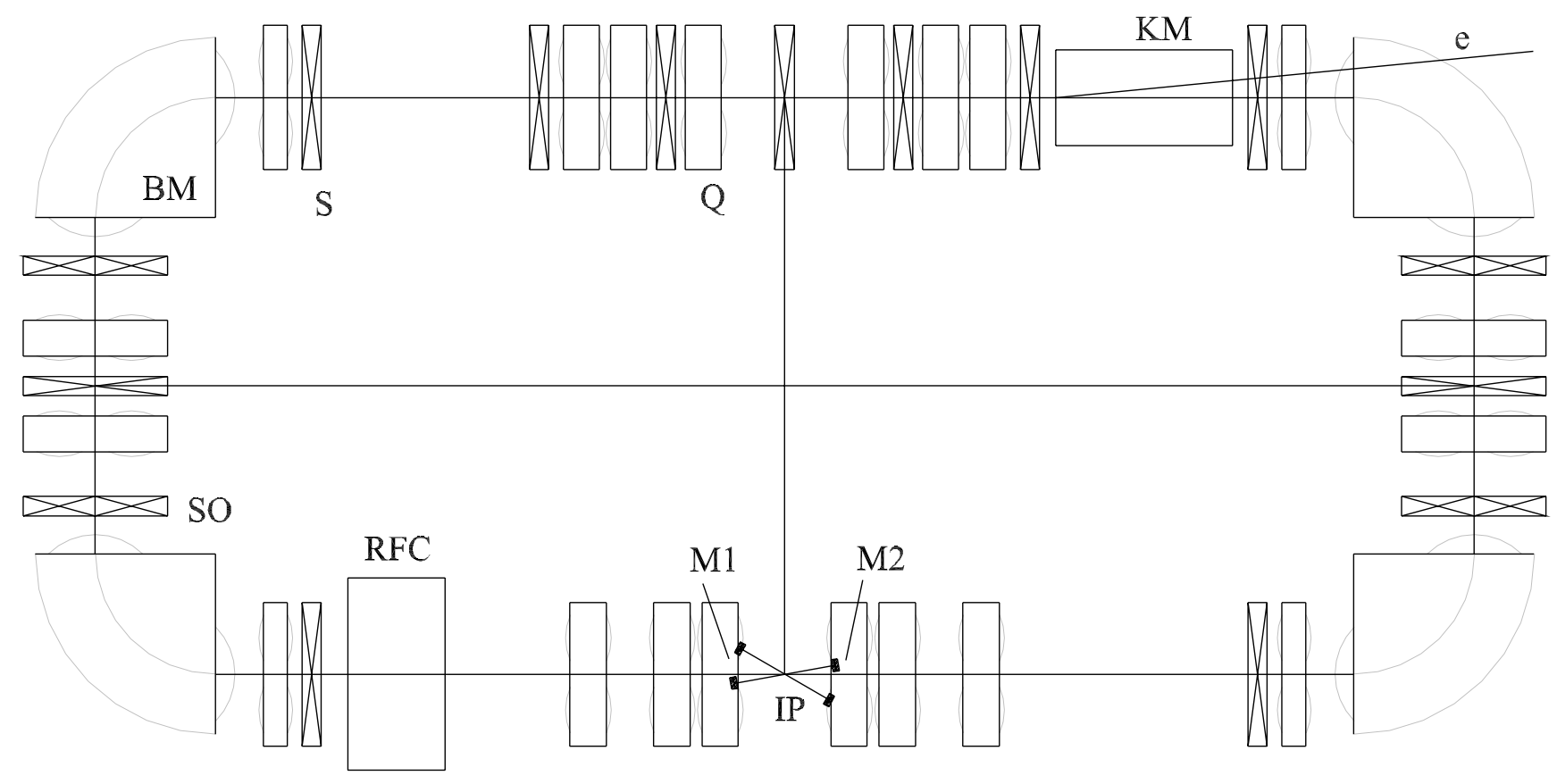

FIG. 1. LESR layout. IP is the interaction point, BM the bending magnets, Q, S, SO the quadrupole, sextupole, and combined sextupole and octupole lenses, correspondingly, RFC the rf cavity, KM the injection kicker magnet, M1-M2 the mirrors of the optical cavities. 


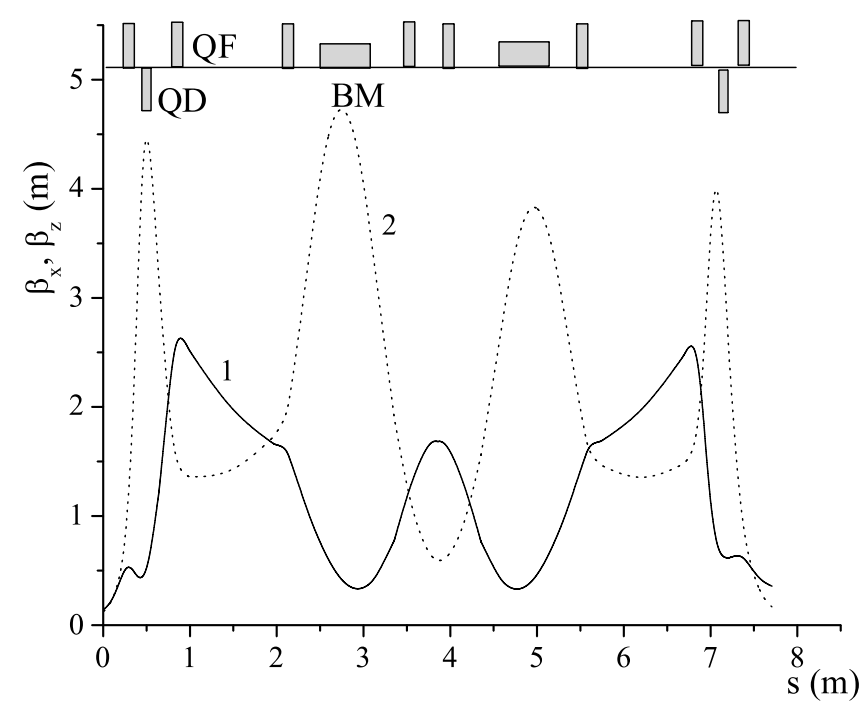

FIG. 2. Horizontal $\beta_{x}(1)$ and vertical $\beta_{z}(2)$ amplitude functions over half of ring lattice.

Fig. 3. We can obtain zero linear dispersion either over both long straight sections or over one of them by controlling of the strength of separated quadrupole in the ring arc. In the first case we obtain the operation mode with a large linear momentum compaction factor $\alpha_{1}=0.078$ (BM mode). In the second case dispersion function $\eta_{1}$ is negative on orbit within one bending of the arc, and we obtain operation mode with decreased momentum compaction factor (LM mode). Dispersion function $\eta_{1}$ in Fig. 3 corresponds to $\alpha_{1}=0.019$. The maximal value of the dispersion function on arc $\eta_{1 \max } \approx 1.2 \mathrm{~m}$, its maximal value on long straight section is equal to $0.3 \mathrm{~m}$.

The dynamic aperture of the storage ring at IP azimuth in operation mode with $\alpha_{1}=0.0096$ is presented in Fig. 4 .

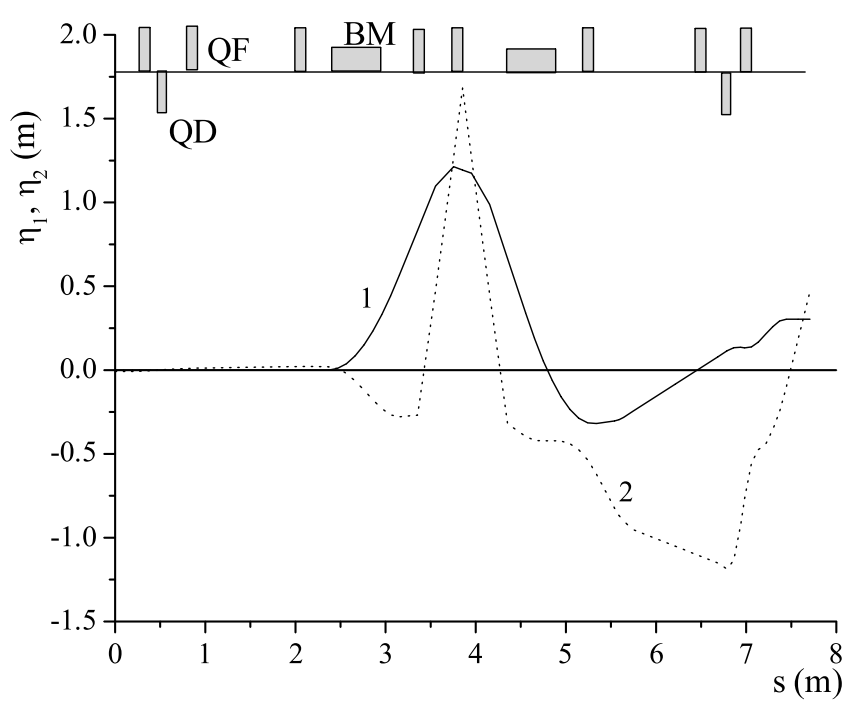

FIG. 3. First order dispersion function $\eta_{1}(1)$ and second one (2) over half of storage ring lattice.

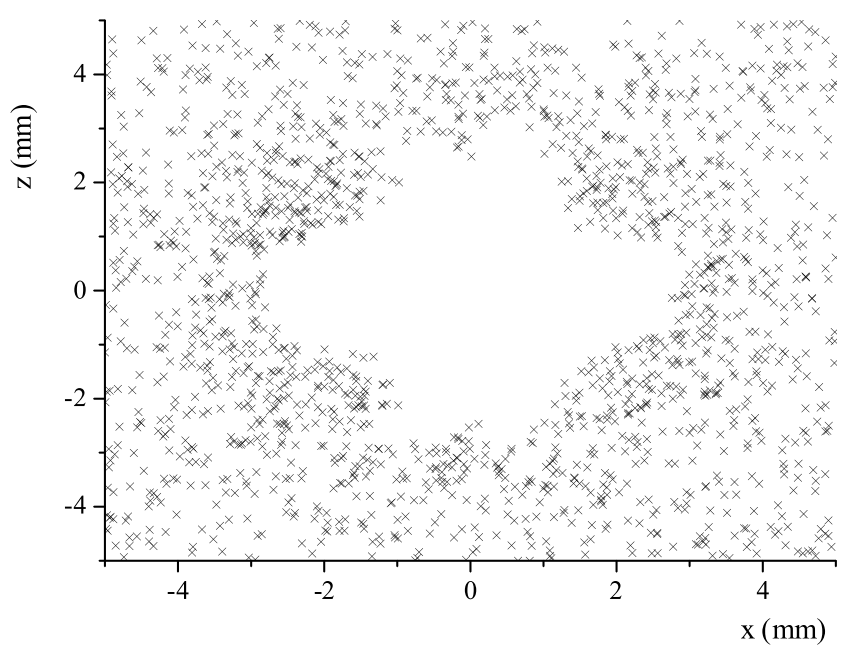

FIG. 4. Dynamic aperture of ring at IP azimuth at initial momentum deviation $\delta_{\text {ini }}=3.5 \%$. Number of particles are 3000 , turns number 50000 . Lost particles are marked by $\times$.

A lot of sextupoles in ring lattice can excite various nonlinear resonances. On the other hand, we can optimize their arrangement and strengths to minimize the effect of resonances and to enlarge DA. Such a procedure was carried out at lattice design using the HARMON module [7]. The dynamic aperture at the azimuths with maximal amplitude functions is about \pm 14 and $\pm 17 \mathrm{~mm}$ in the horizontal and vertical plane, correspondingly. Such DA allows one to keep the intense electron beam with large momentum deviation in the storage ring. For example, the horizontal size of electron bunch because of IBS at IP $\sigma_{x} \approx 0.8 \mathrm{~mm}$ at the electron energy $E_{0}=43 \mathrm{MeV}$, stored bunch current $I_{b}=10 \mathrm{~mA}$ and coupling coefficient $\kappa=0.05$. The dynamic aperture in both the horizontal and vertical plane greatly exceeds the steady-state beam size.

The lattice is very flexible, and it allows to one change the momentum compaction factor over a wide range without betatron detuning and without essential change of the amplitude functions (we can decrease $\eta_{1}$ down to zero and even make it negative). The second order dispersion $\eta_{2}$ is suppressed at IP. It is impossible to solve this problem in BM mode. This statement is illustrated in Figs. 5 and 6 where the trajectories of the particle with large momentum deviation in both operation modes are presented.

In these figures one can see that the particle trajectory in $\mathrm{BM}$ mode depends essentially on the momentum deviation, and this effect causes growth of the effective emittance. Emittance in BM mode is about of 15 times greater than the one in LM mode for simulation parameters. It causes the decreasing of intensity of the scattered beam, as it follows from (4). This effect becomes apparent at high electron beam energy, when the steady-state beam size becomes small due to IBS weakening. Besides, in BM-mode electron beam is slowly exited and may be lost at synchrobetatron resonances if the rf cavity is placed at azimuth with 


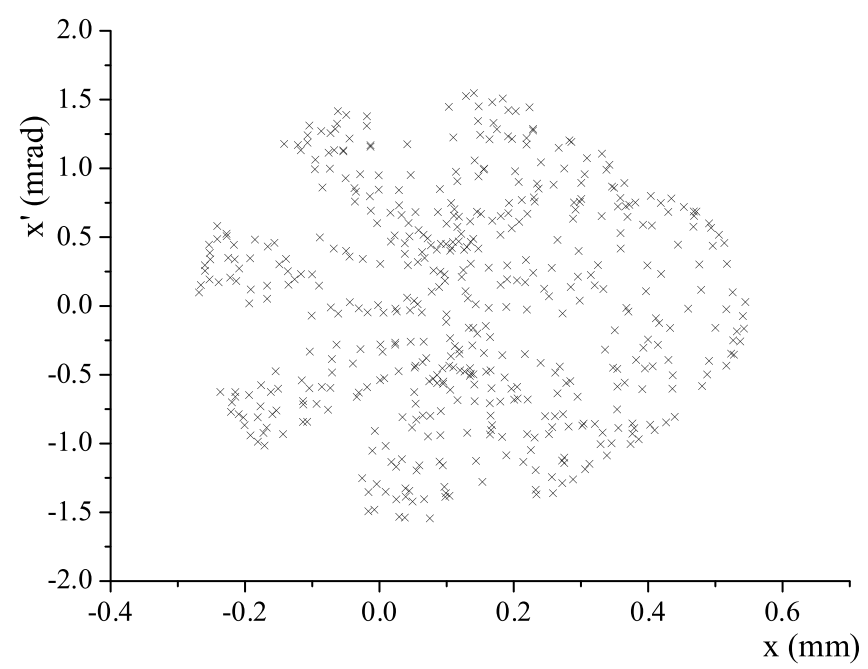

FIG. 5. Horizontal phase trajectory of a particle with large momentum deviation at IP azimuth in operation mode with large momentum compaction factor $\alpha_{1}=0.078$. The initial particle coordinates are $x_{\text {ini }}=z_{\text {ini }}=0.1 \mathrm{~mm}, \quad x_{\text {ini }}^{\prime}=z_{\text {ini }}^{\prime}=s_{\text {ini }}=0$, $\delta_{\text {ini }}=0.01$.

nonzero dispersion. We observed this phenomenon in simulations.

Particle trajectory in LM mode depends insignificantly on momentum deviation. The value of the second order momentum compaction factor is small, $\alpha_{2}=0.38$. As was shown in our beam dynamics simulation, the value of the rf acceptance is greater than $7 \%$ at maximal electron beam energy for such relation between linear and quadratic momentum compaction factors. The dynamics aperture of the storage ring exceeds the physical aperture. Both

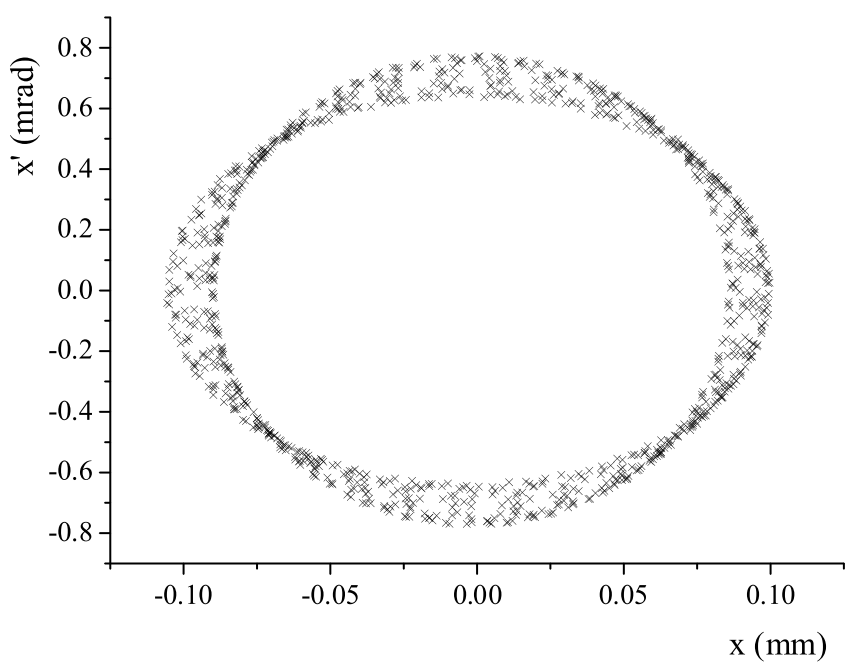

FIG. 6. Horizontal phase trajectory of a particle with large momentum deviation at IP azimuth in the operation mode with low momentum compaction factor $\alpha_{1}=0.019$. The initial particle coordinates are $x_{\text {ini }}=z_{\text {ini }}=0.1 \mathrm{~mm}, x_{\text {ini }}^{\prime}=z_{\text {ini }}^{\prime}=s_{\text {ini }}=0$, $\delta_{\text {ini }}=0.01$. large energy acceptance and DA will allow storing of the intense electron beam and, consequently, obtaining of the intense $\mathrm{x}$ rays.

The MAD lattice deck for the case of the low momentum compaction factor $\alpha_{1}=0.0096$ is presented in Algorithm I (quadrupole and multipole strengths for one of the variants of BM mode with $\alpha_{1} \approx 0.08$ are listed in parentheses). In BM mode the maximal value of the second order dispersion over the ring $\left|\eta_{2 \max }\right| \approx 5.1 \mathrm{~m}$, its value at IP $\eta_{2 I P} \approx-1.25 \mathrm{~m}$. As it was noted above the nonzero dispersion at IP causes considerable growth of the electron beam emittance. It should be noted that designed lattice is optimized for the operation mode with the lowest momentum compaction factor.

\section{PARAMETERS OF X RAYS AND ELECTRON BEAM}

Under CS of the laser photon on relativistic electron, the energy of the scattered $\mathrm{x}$ ray is determined by the expression [12]

$$
\varepsilon_{\gamma}=\frac{1+\beta \cos \varphi}{1-\beta \cos \vartheta} \varepsilon_{\mathrm{las}},
$$

where $\vartheta$ is the angle between vectors of electron and x-ray velocities and $\beta=v / c$ is the ratio of electron and light speeds. One can easily change the energy of scattered photons by changing either the electron energy $(\beta)$, or the collision angle $(\varphi)$, or the observation angle $(\vartheta)$.

As it was noted above, the electron beam energy $E_{0} \approx$ $43 \mathrm{MeV}$ is needed to obtain $\mathrm{x}$ rays for angiographic studies at head-on collision. Unfortunately, the IBS strongly disturbs the electron beam motion at such a low energy and, as a result, the beam emittance quickly grows, and it causes the decreasing of the intensity of CS. The operation mode at steady-state electron beam size is practically impossible because of the low intensity of CS. Our simulations show that under realizable parameters of storage ring and laser system (charge of electron bunch $q_{b}=0.5 \mathrm{nC}$, number of bunches $n_{b}=18$, energy of laser flash stored into optical cavity $w_{\text {las }}=1 \mathrm{~mJ}$ ) the value of the intensity of Compton beam does not exceed $4 \times 10^{11}$ phot/s. It is caused by large steady-state horizontal $\epsilon_{x}$ and longitudinal $\epsilon_{s}$ emittances $\left(\epsilon_{x} \approx 5 \times 10^{-6} \mathrm{~m}, \epsilon_{s} \approx 1.6 \times 10^{-5} \mathrm{~m}\right.$ at coupling coefficient $\kappa=0.05$ and $V_{\text {rf }}=0.3 \mathrm{MV}$ ).

One can obtain angiographic $\mathrm{x}$ rays at electron beam energies $E_{0}>100 \mathrm{MeV}$, when IBS appears to be low. For example, such photons will be generated at the electron beam energy $E_{0}=165 \mathrm{MeV}$ and the collision angle $\varphi=$ $150^{\circ}$. The steady-state emittances will be low in this case $\left(\epsilon_{x} \approx 2.8 \times 10^{-7} \mathrm{~m}, \epsilon_{s} \approx 2.2 \times 10^{-6} \mathrm{~m}\right)$, but the intensity of $\mathrm{x}$-ray photons $n_{\gamma} \approx 1.1 \times 10^{11}$ phot/s will also be low because of the large collision angle, as is evident from (4).

Obviously, at a low electron beam energy the LESR scheme with small collision angle and high repetition 
Algorithm I. The MAD lattice deck for the case of the low momentum compaction factor $\alpha_{1}=$ 0.0096.

title, "LESR, alfa=0.0096"

assign , echo=mad.out ;

! beam definition

be 1 : beam, particle $=$ electron, energy $=0.043$, bunched,

ex $=1.0 \mathrm{~d}-6$, ey $=1.0 \mathrm{~d}-6$, sige $=1.0 \mathrm{~d}-2$,

kbunch $=1$, npart=3.21e9 ;

! elements definition

d 01a : drift, $1=0.097$;

d $02 \mathrm{a}:$ drift, $1=0.025$;

d 03a : drift, $1=0.0995$;

d $04 \mathrm{a}$ : drift, $1=0.100$;

d $04 \mathrm{~b}:$ drift, $1=0.488$;

d 05a : drift, $1=0.100$;

d 06a : drift, $1=0.250$;

d 06b : drift, $1=0.175$;

d $07 \mathrm{a}:$ drift, $\mathrm{l}=0.125$;

d 08a : drift, $1=0.175$;

d $08 b$ : drift, $1=0.250$;

d 09a : drift, $\mathrm{l}=0.100$;

d 10a : drift, $1=0.100$;

d 10b : drift, $1=0.474$;

d 11a : drift, $1=0.023$;

d 12a : drift, $1=0.085$;

d 13a : drift, $\mathrm{l}=0.1275$;

q $01 \mathrm{a}:$ quadrupole, $\mathrm{l}=0.0375, \mathrm{k} 1=35.82489 ;$ !(33.44304)

q $02 \mathrm{a}$ : quadrupole, $\mathrm{l}=0.0375, \mathrm{k} 1=-35.82489 ;$ !(33.44304)

q 03a : quadrupole, $\mathrm{l}=0.0375, \mathrm{k} 1=15.67584 ; !(12.27980)$

q $04 \mathrm{a}:$ quadrupole, $\mathrm{l}=0.025, \mathrm{k} 1=9.00000 ;$ !(9.00000)

q 05a : quadrupole, $\mathrm{l}=0.0375, \mathrm{k} 1=8.68409 ;$ !(7.37370)

q 06a : quadrupole, $\mathrm{l}=0.0375, \mathrm{k} 1=8.68409 ;$ !(7.37370)

q $07 \mathrm{a}:$ quadrupole, $\mathrm{l}=0.025, \mathrm{k} 1=9.00000 ; !(9.00000)$

q 08a : quadrupole, $\mathrm{l}=0.0375, \mathrm{k} 1=21.33293 ;$ !(25.29618)

q 09a : quadrupole, $\mathrm{l}=0.0375, \mathrm{k} 1=-30.27903 ; \mathrm{l}(-33.41186)$

q 10a : quadrupole, $\mathrm{l}=0.0375, \mathrm{k} 1=12.97591 ;$ !(19.29494)

bm 1 : sbend, $\mathrm{l}=0.19635$, angle $=$ pi $/ 8 ., \mathrm{k} 1=-2.400$;

oc 1 : multipole, $\mathrm{k} 31=-61.66 ;$ !(495.0)

oc 2 : multipole, $\mathrm{k} 3 \mathrm{l}=-57.38 ; !(-500.0)$

sc $1:$ multipole, $\mathrm{k} 2 \mathrm{l}=-8.196 ; !(-9.650)$

sc 2 : multipole, $\mathrm{k} 2 \mathrm{l}=5.883 ;$ !(5.970)

sc 3 : multipole, $\mathrm{k} 2 \mathrm{l}=-25.90 ; !(0.290)$

sf 1 : multipole, $\mathrm{k} 2 \mathrm{l}=15.00 ; !(19.27)$

sf 2 : multipole, $\mathrm{k} 2 \mathrm{l}=-8.319 ; !(-0.742)$

sf 3 : multipole, $\mathrm{k} 2 \mathrm{l}=49.83 ; !(-3.488)$

sf 4 : multipole, $\mathrm{k} 2 \mathrm{l}=42.08 ; !(-50.00)$

sd 1 : multipole, $\mathrm{k} 2 \mathrm{l}=-9.972 ; !(-31.72)$

sd 2 : multipole, $\mathrm{k} 2 \mathrm{l}=-23.17 ; !(-6.652)$

sd 3 : multipole, $\mathrm{k} 2 \mathrm{l}=-49.98 ; !(-20.33)$

rfc : rfcavity, type $=r f$, volt $=0.300$, harmon $=36$, lag $=0.500$;

! lines definition

d $01:$ line $=(\mathrm{d} 01 \mathrm{a}, \mathrm{d} 01 \mathrm{a})$

d $02:$ line $=($ d02a, d02a $)$

d $03:$ line $=($ d03a, sc1, d03a $)$

d 04 : line $=($ d04a, sc2, d04b, d04b, sc3, d04a $)$

d $05:$ line $=($ d05a, d05a $)$

d $06:$ line $=($ d06a, sd1, oc1, d06b $)$

d $07:$ line $=($ d07a, sf1, d07a $)$ 
Algorithm I. (Continued.)

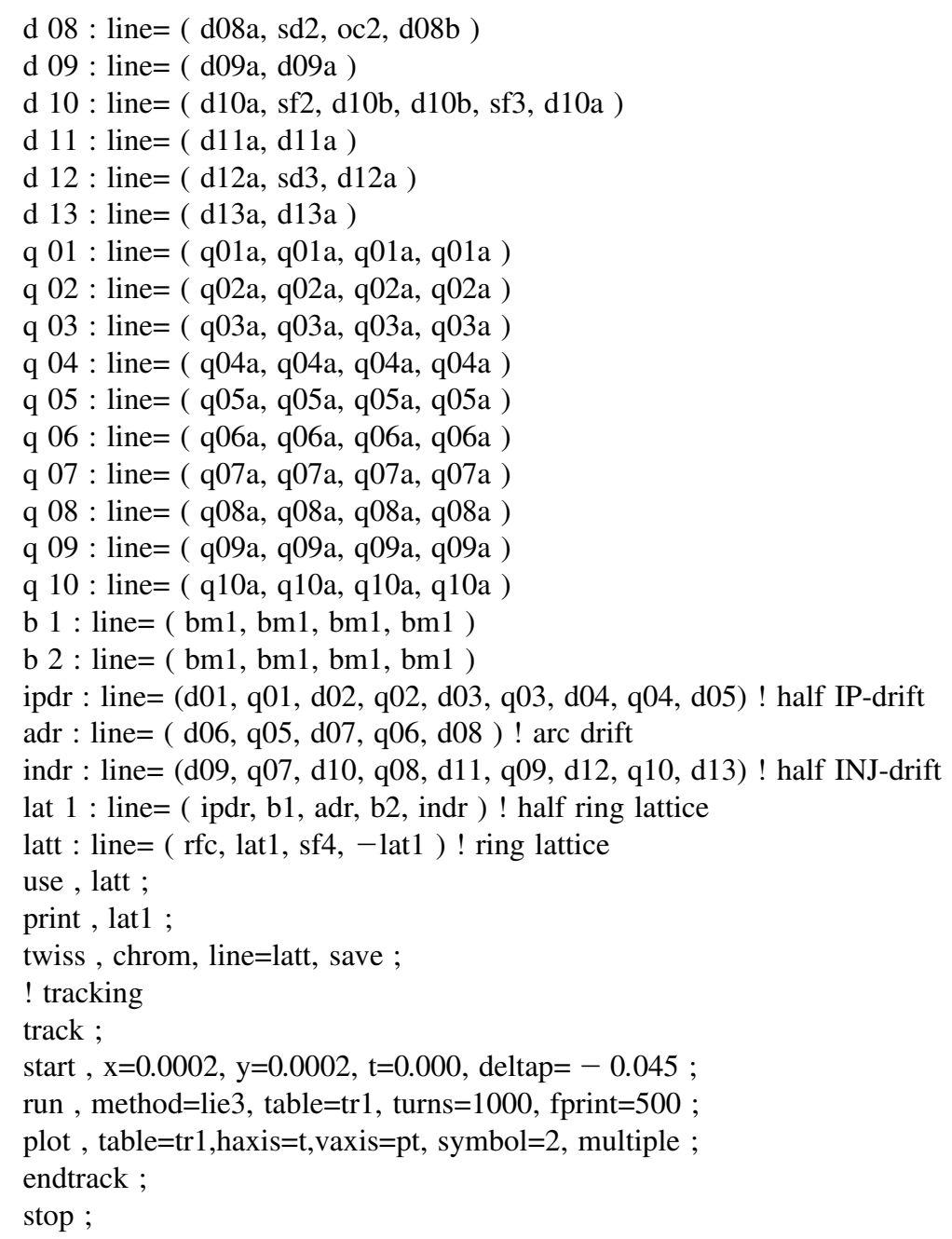

frequency of the injection $f_{\text {inj }}$ of intense, low-emittance electron beam is the most acceptable scheme to resolve this contradiction at state of the art technique [13]. At high injection frequency the electron beam size does not grow essentially during the injection interval, and it allows one to obtain the high average intensity of the x-ray beam. Such a scheme of generation of the angiographic photons is supposed to be used in describing the x-ray source. The computed intensity of the $\mathrm{x}$-ray beam during injection interval for $f_{\text {inj }}=10 \mathrm{~Hz}$ and realizable collision angle $\varphi=10^{\circ}$ is shown in Fig. 7. We simulated CS in the ring lattice by Monte-Carlo method [14].

During the simulation interval because of IBS the horizontal emittance $\epsilon_{x}=10^{-7} \mathrm{~m}$ becomes 10 times as large as the initial one; longitudinal emittance $\epsilon_{s}=7 \times 10^{-6} \mathrm{~m}$ becomes 3 times as large. In these conditions the intensity of Compton scattering quickly decreases. The average intensities during the simulation interval and within the $10 \mathrm{~ms}$ period just after injection are approximately equal to $1.2 \times 10^{12} \mathrm{phot} / \mathrm{s}$ and $1.8 \times 10^{12} \mathrm{phot} / \mathrm{s}$.

It should be recalled that the proposed lattice is appropriate for steady-state operation modes all over the electron energy range. Unfortunately, such an operation is impossible at low energies because of insufficient laser flash energy, stored into the optical cavity at state of the art laser systems.

The collimated spectrum of Compton radiation within $1 \mathrm{mrad}$ collimation angle for the above described conditions is presented in Fig. 8. The spectral brightness under laser beam size $\sigma_{\text {las }}=40 \mu$ is approximately equal to $4 \times$ $10^{12} \mathrm{phot} /\left(\mathrm{s} \mathrm{mm}^{2} \mathrm{mrad} 0.1 \% \mathrm{BW}\right)$ and such brightness allows one to begin angiographic studies.

Most biological studies require $\mathrm{x}$ rays with photon energy within the range of $5 \leq \varepsilon_{\gamma} \leq 16 \mathrm{keV}$, and the longterm stability of Compton beam intensity is very important for some of them. At a head-on collision we need to use the electron beam with energies within the range of $18<E_{0}<$ $30 \mathrm{MeV}$ in order to generate such an x-ray beam. The steady-state operation mode of the storage ring with intensive electron beam is impossible at such low energies because of IBS. To meet the requirements of the biological studies one can use Compton scattering at a large collision angle. For example, at $\varphi=150^{\circ}$ the energy of the electron beam over the range $70<E_{0}<120 \mathrm{MeV}$ is needed $\left(150^{\circ}\right.$ 


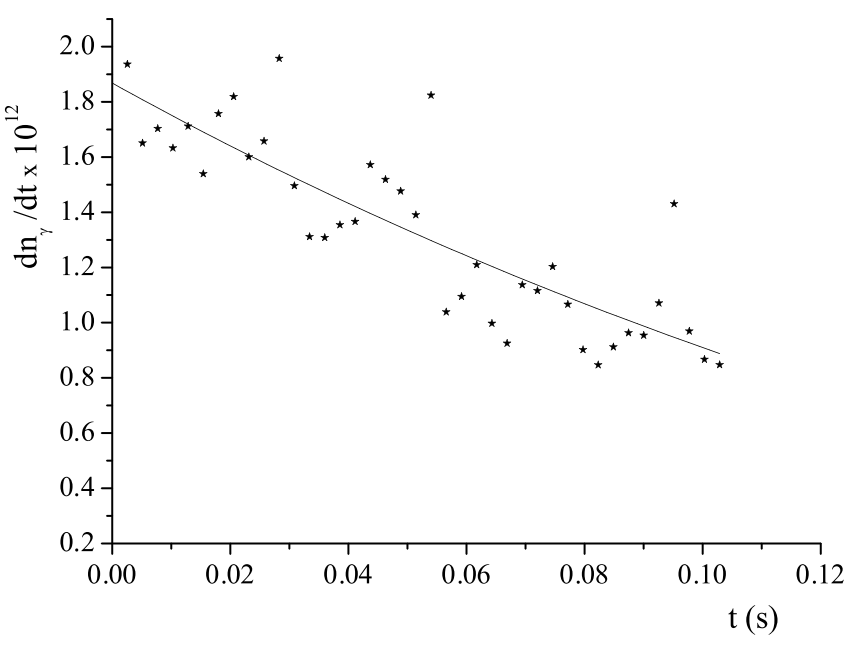

FIG. 7. Intensity of $\mathrm{x}$ rays vs time. The number of electrons per bunch is $n_{e}=3.2 \times 10^{10}(0.5 \mathrm{nC} /$ bunch $)$, the number of bunches $n_{b}=18$, stored laser flash energy $w_{\text {las }}=1 \mathrm{~mJ}$.

collision is supposed to be used in the designed lattice). Of course, $x$-ray intensity at such a collision angle will be much less than the one at a small collision angle, $n_{\gamma} \approx$ $10^{11} \mathrm{phot} / \mathrm{s}$. Nevertheless, this intensity meets the requirements of the biological experiments. Besides, $x$-ray intensity will be very stable because the electron beam with steady-state parameters will be used.

The collimated spectrum of the photons for biological studies with maximal energy $\varepsilon_{\gamma \max } \approx 6.7 \mathrm{keV}$ is presented in Fig. 9. Total Compton beam intensity $n_{\gamma} \approx 4 \times$ $10^{10} \mathrm{phot} / \mathrm{s}$, number of photons within the collimation angle $\alpha_{\text {col }}=1 \mathrm{mrad}$ is $n_{\gamma \mathrm{col}} \approx 1.1 \times 10^{9} \mathrm{phot} / \mathrm{s}$, spectrum width (FWHM) is approximately equal to $10 \%$. Under laser beam waist $\sigma_{\text {las }}=40 \mu$, the spectral bright-

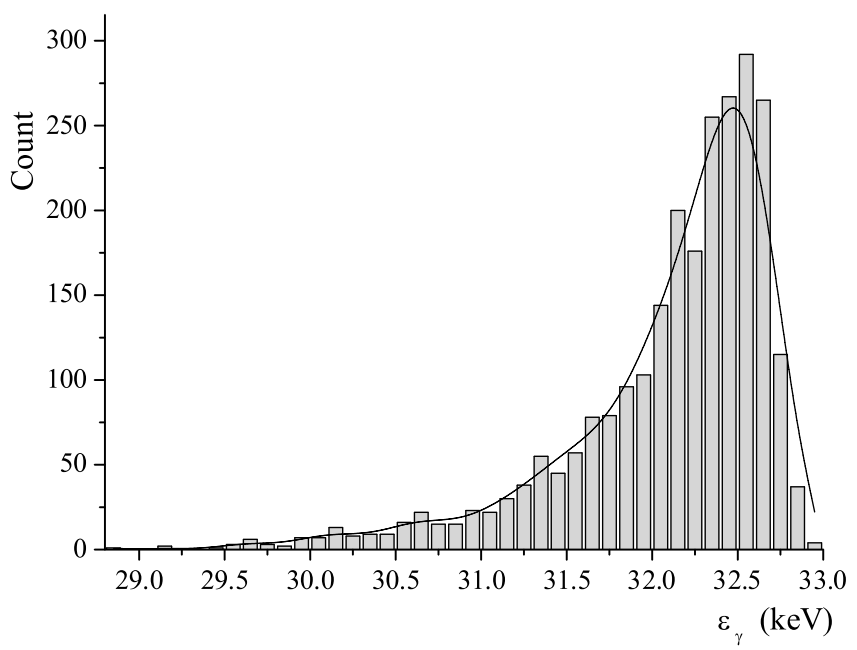

FIG. 8. Spectrum of scattered photons within $1 \mathrm{mrad}$ collimation angle. Electron beam energy $E_{0}=43 \mathrm{MeV}$, collision angle $\varphi=10^{\circ}$, initial emittances of injected beam $\epsilon_{x}=\epsilon_{z}=10^{-7} \mathrm{~m}$, $\epsilon_{s}=7 \times 10^{-6} \mathrm{~m}$. ness at maximum of spectral density $B \approx 1 \times$ $10^{11} \mathrm{phot} /\left(\mathrm{s} \mathrm{mm}^{2} \operatorname{mrad} 0.1 \% \mathrm{BW}\right)$.

Comparing the spectra in Fig. 8 with that in Fig. 9, one can see that at $E_{0}=43 \mathrm{MeV}$ spectrum width $\sigma_{\gamma}=$ $\Delta \varepsilon_{\gamma} / \varepsilon_{\gamma} \approx 3 \%$ is much less than $\sigma_{\gamma} \approx 10 \%$ at electron beam energy $E_{0}=75 \mathrm{MeV}$. These spectra illustrate the effect of divergence of the electron beam on spectrum of photons generated under CS. Spectrum width essentially depends on the relation of beam divergence $\sigma_{x \prime}$ and characteristic angle $\alpha_{c}=1 / \gamma$ because the change in the trajectory angle is equivalent to the change in the observation angle [see Eq. (6)]. During simulation interval $\sigma_{x /}$ at $E_{0}=$ $43 \mathrm{MeV}$ changes from $\sigma_{x \prime} \approx 0.9 \mathrm{mrad}$ to $\sigma_{x l} \approx 3 \mathrm{mrad}$, so, $\sigma_{x /} \ll \alpha_{c} \approx 12 \mathrm{mrad}$. Under such conditions, the spectrum width increases insignificantly comparably to the ideal collimated spectrum.

On the contrary, beam divergence in simulation at $E_{0}=$ $75 \mathrm{MeV}$ is comparable to characteristic angle because we use the electron beam with the steady-state emittances: $\sigma_{x \prime} \approx 3.3 \mathrm{mrad}, \alpha_{c} \approx 6.8 \mathrm{mrad}$. It leads to significant widening of the ideal collimated spectrum of the scattered photons.

Partial spectrum widths $\sigma_{\gamma} \approx 2 \delta_{\text {tot }}$ [5] caused by the energy spread in electron beam are negligible in both spectra.

In order to generate hard $\gamma$ quanta the $10^{\circ}$ collision of laser photons and a high-energy electron beam with steadystate parameters will be used in described the lattice. At a high electron beam energy the steady-state emittances are low, consequently, we will be able to reach the high intensity of $\mathrm{x}$ rays (in the proposed lattice $n_{\gamma} \approx 5 \times 10^{12} \mathrm{phot} / \mathrm{s}$ for the above described parameters of electron and laser beams). The maximal energy of $\gamma$ quanta is $\varepsilon_{\gamma \max } \approx$ $900 \mathrm{keV} \quad\left(\varepsilon_{\text {las }}=1.164 \mathrm{eV}\right.$, electron energy $E_{0}=$

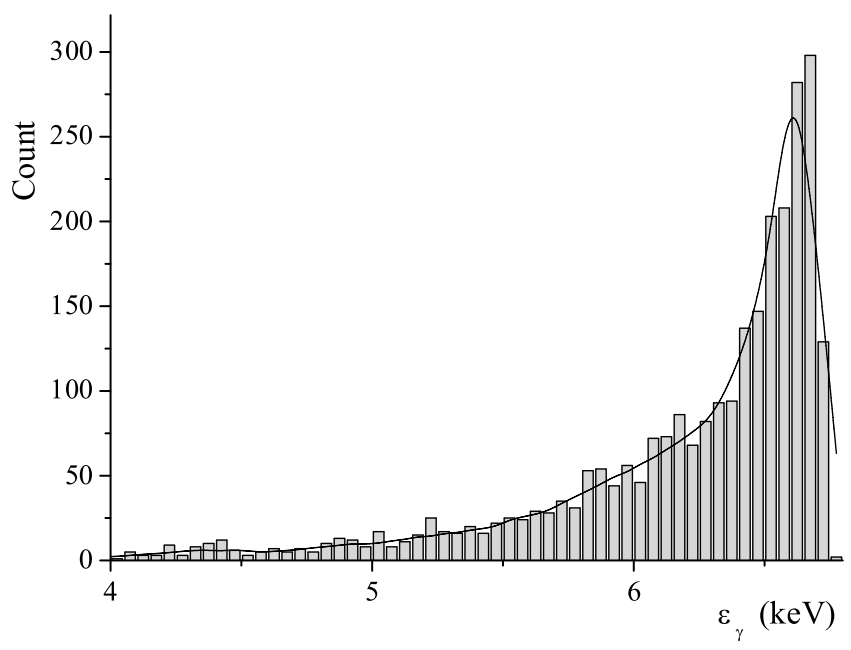

FIG. 9. Spectrum of scattered photons within $1 \mathrm{mrad}$ collimation angle. Electron beam energy $E_{0}=75 \mathrm{MeV}$, collision angle $\varphi=150^{\circ}$, steady-state emittances of electron beam $\epsilon_{x}=1.5 \times$ $10^{-6} \mathrm{~m}, \epsilon_{s}=6.6 \times 10^{-6} \mathrm{~m}$. 


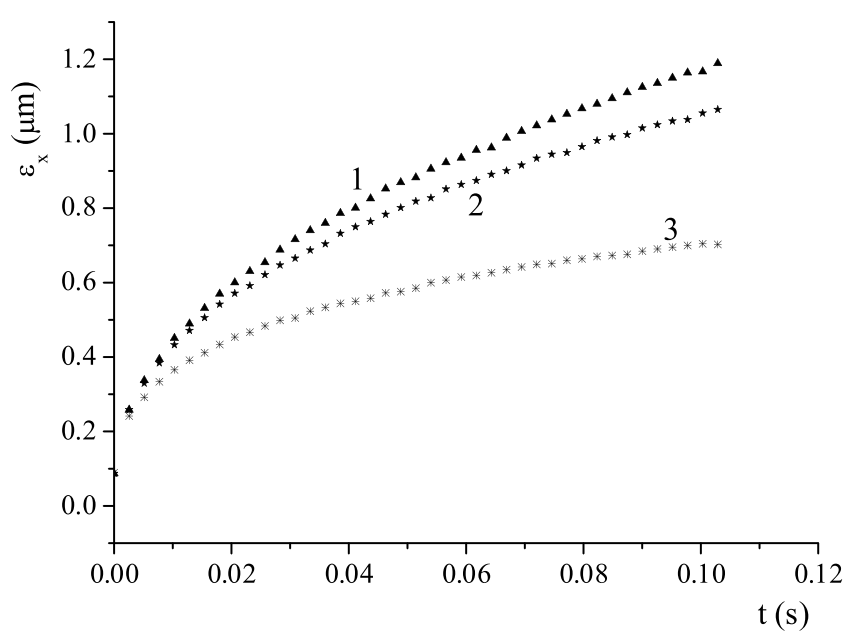

FIG. 10. Behavior of horizontal emittance for different stored energy of laser flash. Curves $1,2,3$ correspond to $w_{\text {las }}=1,10$, $100 \mathrm{~mJ}$.

$225 \mathrm{MeV})$. By means of the "green laser" $\left(\varepsilon_{\text {las }}=\right.$ $2.328 \mathrm{eV}$ ) we will be able to obtain the energy of $\gamma$ quanta about of $1.8 \mathrm{MeV}$. For example, such $\mathrm{x}$ rays are used for neutron generation in a beryllium target.

In the closing of this section, the dependencies of horizontal emittance of the electron beam on time for various stored energies of laser flash as the illustration of the laser cooling of electron beam are presented in Fig. 10. Of course, even $w_{\text {las }}=1 \mathrm{~mJ}$ stored into the optical cavity is a challenge parameter, let alone $w_{\text {las }} \sim 100 \mathrm{~mJ}$ [15], but such parameters of laser systems may be realized in the future. In any case, in Fig. 10 one can see the level of those parameters needed for laser cooling experiments.

\section{SUMMARY}

The problems of the electron beam dynamics associated with large beam energy spread caused by intensive Compton scattering are solved in the proposed lattice of the laser-electron storage ring. $X$ rays over the energy range $6 \leq \varepsilon_{\gamma} \leq 900 \mathrm{keV}$ with long-term stable intensity up to $10^{13} \mathrm{phot} / \mathrm{s}$ will be generated under realizable parameters of the injector, storage ring and laser system. The maximum allowed intensity of the Compton beam limited by energy acceptance of the storage ring is not less than $10^{15} \mathrm{phot} / \mathrm{s}$ over all energy range.

The problems of instability of electron beam in LESR, limiting the intensity of stored beam, are not discussed in this paper. The study of such instabilities, evaluations of the coupling impedances of the chamber, etc., requires much effort and will be the object of further investigation. Nevertheless, preliminary estimations carried out [16,17] show the possibility of storing the current $I_{b} \sim 10 \mathrm{~mA}$ per bunch (the number of particles per bunch $n_{e b} \approx 3 \times 10^{9}$ ) without essential bunch lengthening, because the value of
TABLE I. Main parameters of the storage ring and parameters of electron and Compton beams.

\begin{tabular}{lc}
\hline \hline \multicolumn{1}{c}{ Parameter } & Value \\
\hline Circumference, $m$ & 15.418 \\
Energy range, $\mathrm{MeV}$ & $40-225$ \\
Betatron tunes & $3.15 ; 2.08$ \\
Amplitude functions at IP, m & $0.14 ; 0.12$ \\
Linear momentum compaction factor & $0.01-0.078$ \\
$R F$-acceptance, $\%$ & $\geq 7$ \\
$R F$-frequency, MHz & 700 \\
$R F$-voltage, MV & 0.3 \\
Harmonics number & 36 \\
Electron bunch current, mA & 10 \\
Stored energy of laser flash, mJ & 1 \\
Collision angle, degrees & $10 ; 150$ \\
X-rau energy $\left(\varepsilon_{\text {las }}=1.16 \mathrm{eV}\right), \mathrm{keV}$ & $6-900$ \\
Maximum intensity of $\mathrm{x}$ rays, phot/s $\left(w_{\text {las }}=1 \mathrm{~mJ}\right)$ & $\mathrm{Up}$ to $10^{13}$ \\
\hline \hline
\end{tabular}

the momentum compaction factor is large enough in assumed operation modes $0.01 \lesssim \alpha_{1} \lesssim 0.02$. We cannot use the very low momentum compaction factor because in the proposed lattice the $\mathrm{rf}$ acceptance is quickly decreased at $\alpha_{1}<0.01$ and the storage ring becomes inappropriate for Compton scattering.

The main parameters of the storage ring and parameters of electron and Compton beams are presented in Table I.

\section{ACKNOWLEDGMENTS}

The author is grateful to Dr. E. Bulyak, Dr. I. Karnaukhov, Dr. A. Mytsykov, Dr. A. Shcherbakov, and Dr. A. Zelinsky of NSC KIPT for help in calculations and helpful discussions, as well as to Dr. J. Urakawa of KEK and Dr. N. Sasao of Kyoto University for detailed discussions of possible operation modes of the storage ring and laser system. This work was partially supported by NATO Grant No. SfP 977982

[1] Z. Huang and R. D. Ruth, Phys. Rev. Lett. 80, 976 (1998).

[2] R. J. Loewen, Ph.D. thesis, Stanford, 2003.

[3] E. Bulyak et al., Nucl. Instrum. Methods Phys. Res., Sect. A 487, 241 (2002).

[4] J. Urakawa et al., Nucl. Instrum. Methods Phys. Res., Sect. A 532, 388 (2004).

[5] E. Bulyak, in Proceedings of the European Particle Accelerator Conference, Lucerne, 2004 (EPS-AG, Lucerne, 2004).

[6] E. Bulyak and V. Skomorokhov, in Proceedings of the European Particle Accelerator Conference, Lucerne, 2004 (Ref. [5]).

[7] M. Donald, Rutherford Laboratory Report No. RL-76-052, 1976.

[8] F. C.Iselin, CERN Report No. CERN/SL/92, 1992. 
[9] C. Pellegrini and D. Robin, Nucl. Instrum. Methods Phys. Res., Sect. A 301, 27 (1991).

[10] L. Lin and C. E. da Silva, Nucl. Instrum. Methods Phys. Res., Sect. A 329, 9 (1993).

[11] J.D. Bjorken and S. K. Mtingwa, Part. Accel. 13, 115 (1983).

[12] A. Akhiezer and V. Berestetsky, Quantum Electrodynamics (Nauka, Moscow, 1969).

[13] J. Urakawa (private communication).
[14] P. Gladkikh and A. Zelinsky, in Proceedings of the European Particle Accelerator Conference, Lucerne, 2004 (Ref. [5]).

[15] M. Nomura et al., in Proceedings of the European Particle Accelerator Conference, Lucerne, 2004 (Ref. [5]).

[16] K. Y. Ng, FNAL Report No. FERMILAB-Conf-96/378, 1996.

[17] B. Zotter and F. Sacherer, CERN 77-13, 1977. 